\title{
Dependence of the GRAPES-3 EAS particle density and trigger rate on atmospheric pressure and temperature
}

M. Zuberi ${ }^{* a b}$, S. Ahmad ${ }^{a c}$, K.P. Arunbabu ${ }^{a b}$, A. Chandra ${ }^{a b}$, S.R. Dugad ${ }^{a b}$, S.K. Gupta $^{a b}$, B. Hariharan ${ }^{a b}$, Y. Hayashi ${ }^{a d}$, P. Jagadeesan ${ }^{a b}$, A. Jain ${ }^{a b}$, V.B. Jhansi ${ }^{a b}$, S. Kawakami $^{a d}$, H. Kojima ${ }^{a e}$, P.K. Mohanty ${ }^{a b}$, S.D. Morris ${ }^{a b}$, P.K. Nayak ${ }^{a b}$, A. Oshima ${ }^{a f}$, B.S. Rao ${ }^{a b}$, L.V. Reddy ${ }^{a b}$, S. Shibata ${ }^{a f}$

${ }^{a}$ The GRAPES-3 Experiment, Cosmic Ray Laboratory, Raj Bhavan, Ooty 643001, India

${ }^{b}$ Tata Institute of Fundamental Research, Mumbai 400005, India

${ }^{c}$ Aligarh Muslim Univeristy, Aligarh 202002, India

${ }^{d}$ Graduate School of Science, Osaka City University, Osaka 558-8585, Japan

${ }^{e}$ Faculty of Engineering, Aichi Insitute of Technology, Toyota City, Aichi 470-0392, Japan

${ }^{f}$ College of Engineering, Chubu University, Kasugai, Aichi 487-8501, Japan

E-mail: meeran.zuberihep@gmail.com

The GRAPES-3 experiment, a high density extensive air shower (EAS) array located at Ooty, India is designed for high precision measurements of cosmic ray energy spectrum and nuclear composition in energy range of $10^{13}-10^{16} \mathrm{eV}$. It consists of tightly packed $1 \mathrm{~m}^{2}$ area 400 plastic scintillation detectors covering an effective area of $25,000 \mathrm{~m}^{2}$. The trigger rate and particle density measured by EAS array shows strong correlation with the atmospheric parameters such as the pressure, and temperature. By employing linear regression, and Fast Fourier Transform techniques, the pressure and temperature coefficients for the dependence of trigger rate and particle density, respectively were obtained. Thereafter, by applying corrections for these effects the contribution of atmospheric effects was largely eliminated in the EAS data. These corrections are expected to result in a better measurement of EAS parameters which will be presented during the conference.

35th International Cosmic Ray Conference - ICRC2017

10-20 July, 2017

Bexco, Busan, Korea

${ }^{*}$ Speaker. 


\section{Introduction}

The physical nature of the cosmic ray variations in different time scales are the important areas to get better understanding in astroparticle physics. Specifically the study of cosmic ray modulation is a prime tool to describe the variable conditions in the atmosphere. The change in the atmospheric pressure and temperature can produces a significant variations in ground based observations. Thus, it is necessary to take an account of these local effects to get better measurements of shower parameters [1]. As expected, at Ooty, the diurnal variations and semi-diurnal periodicity is seen in temperature and pressure respectively (as shown in Fig. 1a). The temperature variation is due to solar heating, wheres, the pressure periodicity is mainly due to the complex interaction of solar heating and the non linear response of the upper atmosphere to this forcing, causes a dominant 12 $\mathrm{h}$ oscillations in pressure at near equatorial regions with an amplitude of $\sim 1 \mathrm{hPa}$, which rapidly decreases at higher latitudes [2-4].

Due to near equatorial region $\left(11.4^{\circ} \mathrm{N}\right)$, the upper atmospheric temperature variation is found to be relatively small at the GRAPES-3 experiment. However, the particle density and the trigger rate shows the periodic variations due to change in the ground level temperature which affects the photo cathode of PMT and shows a significant dependence on surrounding temperature. According to Young [5], the typical temperature coefficient for $\mathrm{Sb}-\mathrm{Cs}$ tubes of about $-0.7 \% /{ }^{\circ} \mathrm{C}$. Moreover Leo [6] also noted down a change of $-0.5 \% /{ }^{\circ} \mathrm{C}$ with rising temperature in the range between $25^{\circ} \mathrm{C}$ to $50^{\circ} \mathrm{C}$ due to changes in the Fermi levels and resistance in the cathode and a few hundredths of a percent for the dynodes. However, both Young and Leo mentioned that these values may vary not only in different type of PMTs, but also within similar PMTs. The temperature effect has been studied for all the plastic scintillation detectors which will be discussed in great details in section 3.1 .

The effect of pressure on cosmic ray flux has been known from a long time. Unlike to the temperature effect, the pressure effect is purely atmospheric, which is mainly due to change in mean free path of cosmic ray particles in the atmosphere. The increase in atmospheric pressure gives rise to the atmospheric air density, which cause a decrease in mean free path of the particles.Hence, the absorption probability of low energy showers in the atmosphere becomes more prominent which leads to deficit in ground level particle density and vice versa. Many studies were carried out to find the pressure coefficient of extensive air showers (EAS). Daudin [7] shows the pressure coefficient value is $(-0.75 \pm 0.01) \% / \mathrm{mb}$ for EAS of primary energy $\approx 10^{14} \mathrm{eV}$ primary energies EAS. In section 3.2, we have studied the atmospheric pressure effect on GRAPES-3 EAS data. The effects on trigger rate will be discussed in section 4 and the results will be discussed in section 5 .

\section{The GRAPES-3 Experiment}

The GRAPES-3 array, located in Ooty, India $\left(11.4^{\circ} \mathrm{N}, 76.7^{\circ} \mathrm{E}\right.$, and $2200 \mathrm{~m}$ above $\left.\mathrm{msl}\right)$ has been designed to study primary cosmic ray energies and its compositions in the range of $10^{13}-10^{16} \mathrm{eV}$. It consists of tightly packed 400 plastic scintillation detectors with the inter-detector separation of $8 \mathrm{~m}$ arranged in hexagonally symmetrical pattern as shown in Fig.1b. Each detector consists of 4 blocks of plastic scintillators, each $5 \mathrm{~cm}$ thick and $50 \times 50 \mathrm{~cm}^{2}$ in area,covers the effective area of $1 \mathrm{~m}^{2}[4,9]$. In addition to that,GRAPES-3 has a highly sensitive large area $\left(560 \mathrm{~m}^{2}\right)$ tracking muon 
telescope in the North-West region of the array with the energy threshold of $\sec (\theta) \mathrm{GeV}$ for muons. It consists of 3712 proportional counters which are equally distributed in 16 different modules(as represented by squares in Fig. 1b) having an area of $35 \mathrm{~m}^{2}$ each [8].

The GRAPES-3 experiment uses a two level trigger system named as Level-0 and Level-1. A three line coincidence is used to generate Level-0 trigger with low energy threshold. However, because of unrestrictive nature of trigger, expectedly 3-line trigger selects an ample number of small as well as very large showers. In order to overcome this difficulty an additional condition was imposed on the shower selection which demands at least 10 out of trigger participating detectors should have triggered the discriminators within $1 \mu \mathrm{sec}$ named as Level-1 or shower trigger, the technical details are discussed elsewhere [4]. The present shower trigger rate of GRAPES-3 experiment is $\sim 42 \mathrm{~Hz}$. All the analysis in this paper has been done by using year 2014 data.

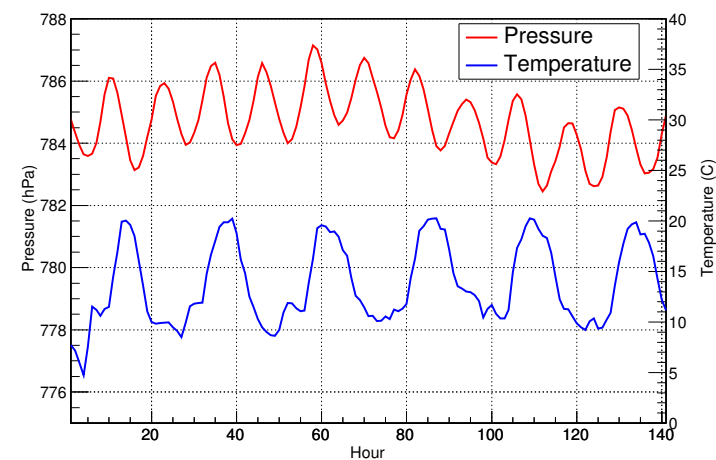

(a) Pressure and temperature variations at GRAPES-3

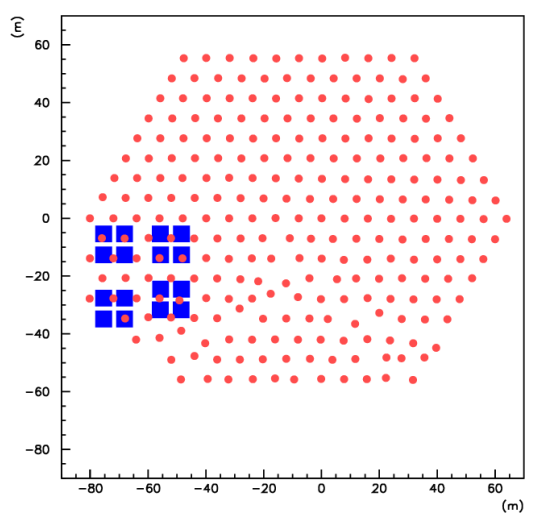

(b) Schematic of the GRAPES-3 EAS array

Figure 1

\section{Atmospheric effects on particle density}

The plastic scintillation detectors are sensitive for both the electromagnetic and the muonic components of the EAS, which are affected by the variation in atmospheric pressure $(\mathrm{P})$ and the air density $(\rho)$. The photomultiplier tube (PMT), an integral part of a plastic scintillation detectors had a high temperature sensitivity. The GRAPES-3 experiment uses an ET Enterprises ET9807B $51 \mathrm{~mm}$ PMT, with a bialkali photo cathode and 12 stages $\mathrm{Be}-\mathrm{Cu}$ dynodes with a maximum gain of $10^{6}$. The temperature coefficient of the Be-Cu as well as Sb-Cs PMTs as specified by the manufacturer is $-0.5 \% /{ }^{\circ} \mathrm{C}$ [11]. The plastic scintillation detectors at the GRAPES-3 EAS array operate at comparably close to room temperature. During winter (Nov-Feb), the daily temperature variation is observed to be $\pm 10{ }^{\circ} \mathrm{C}$, which cause a measurable change in the detectors output.

In this analysis we have used the temperature data recorded by VINTAGE PRO-2, a weather station unit manufactured by DAVIS Instruments located at the center of the array and the atmospheric pressure was measured by two independent digital barometers with the accuracy of $0.1 \mathrm{hPa}$. A self-consistent calibration method [10] has been used to combine data from both units to get an uninterrupted pressure data stream during the analysis period. The Fast Fourier Transform (FFT) technique has been used to obtain the pressure and temperature coefficients of the detectors. 


\subsection{Temperature effect on particle density}

Power spectrum of particle density and temperature obtained using FFT is shown in Fig. 2a (I) and Fig. 2a (II). Both the data sets show a dominant peak at 1 cycle per day (cpd), however former shows one more dominant peak at $2 \mathrm{cpd}$ which is expected due to pressure effect, details will be discussed in next section 3.2. We have designed a band pass filter to select the frequency centered at $1 \mathrm{cpd}$ from both the data sets and then performed Inverse Fast Fourier Transform (IFFT) on the filtered frequency components to convert them back into the time series data. The resultant variations in IFFT data are shown in Fig. $2 b$ for particle density and temperature. The particle density seems to have anti-correlation with temperature, with some time lag. As discussed earlier, the temperature data was measured at one place whereas the detectors are spread over a large area, furthermore the aluminum cover provides the thermal insulation to the detectors from the surrounding. So, the change in the outside temperature gradient took some time to propagate through the insulation to have effects on PMT. Both these factors contribute and produce the time lag was calculated by shifting the temperature data $\pm 3 \mathrm{hrs}$ in the steps of $5 \mathrm{~min}$ with respect to particle density.The correlation coefficient was calculated for each shift and plotted as a function of lag in order to get the maximum lag. Fig. 3a shows the lag plot for detector no. 286 where the observed lag is $\sim 80 \mathrm{~min}$ for -0.96 maximum correlation coefficient.

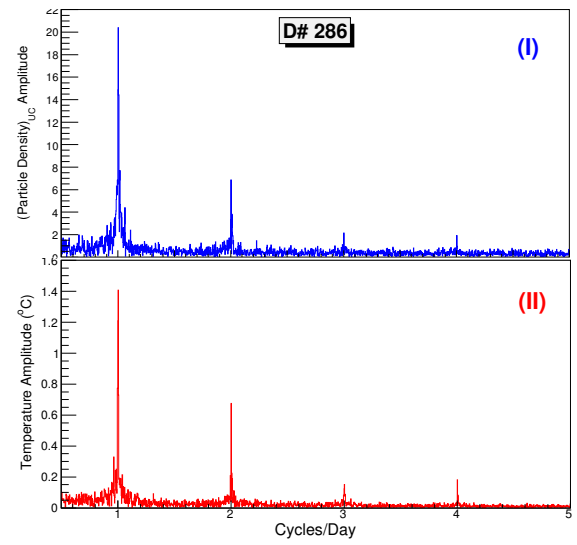

(a) Power spectrum of particle density for Det.286 (I) and temperature (II)

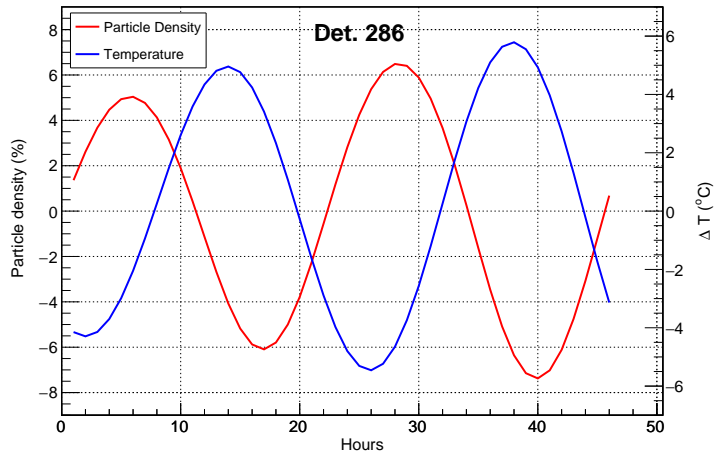

(b) Particle density for Det.286 and temperature variation

Figure 2

The temperature effect is experimentally determined by Eqn. 3.1.

$$
\left(\frac{\Delta I}{I}\right)_{U C}=\beta_{T} \cdot \Delta T
$$

The left hand side of the Eqn. 3.1 is the normalized deviation of the uncorrected particle density and on the right hand side $\Delta \mathrm{T}$ is the temperature deviation from its mean value and $\beta_{T}$ is the temperature coefficient. A clear anti-correlation has been observed between $\left(\frac{\Delta I}{I}\right)_{U C}$ and $\Delta \mathrm{T}$. The value of $\beta_{T}$ has been calculated for all plastic scintillation detectors with the mean value of $0.65 \pm 0.01 \% /{ }^{\circ} \mathrm{C}$ as shown in Fig. 3b. However, the individual detector's temperature coefficients have been used to correct the particle density data for temperature effect. 


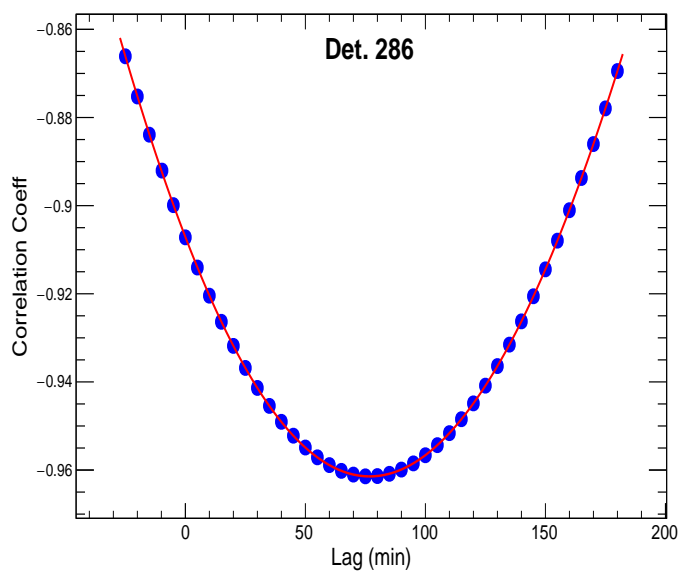

(a) Correlation coefficient and lag variation for Det.286

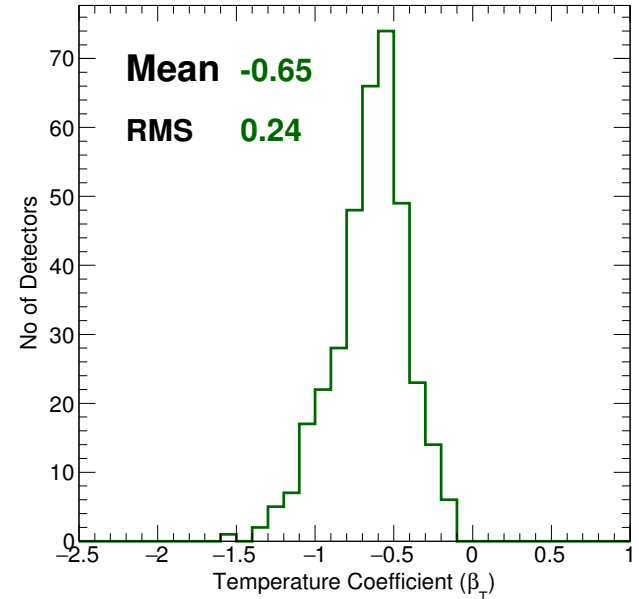

(b) Distribution of temperature coefficients for all plastic scintillation detectors

Figure 3

\subsection{Pressure effect on particle density}

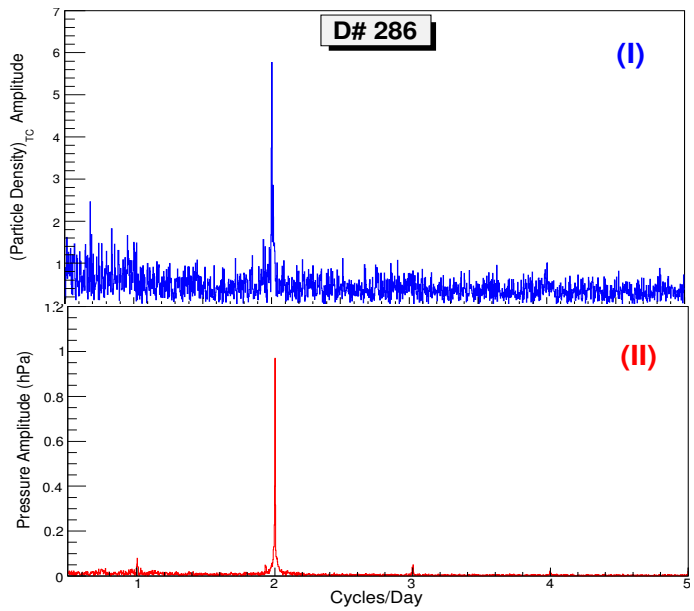

(a) Power spectrum of temperature corrected particle density for Det.286 (I) and pressure (II)

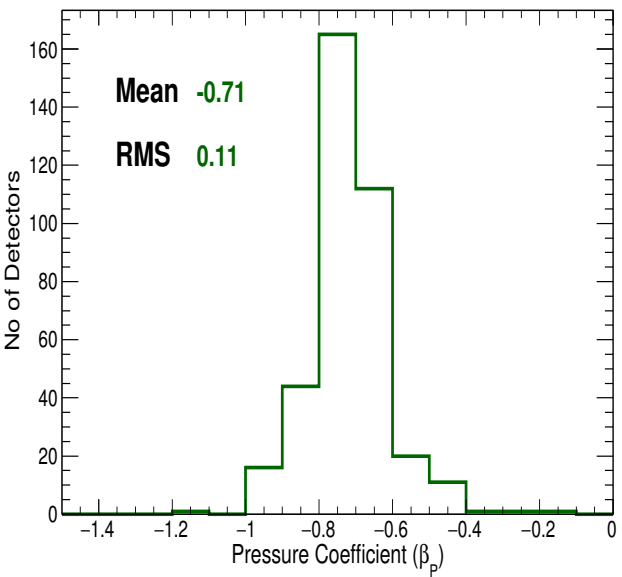

(b) Distribution of pressure coefficients for all plastic scintillation detectors

Figure 4

In order to study the barometric pressure effect, hourly pressure and temperature corrected particle density data have been used. The technique which was discussed in previous section was used to calculate the pressure coefficient. Fig. 4a(I) and Fig. 4a(II) shows the power spectrum of temperature corrected particle density and pressure data respectively. The dominant peak at 2 cpd filtered by using a band pass filter centered at the same frequency component. As expected, the IFFT shows anti-correlation without any time lag. The pressure coefficient determined by the following Eqn. 3.2. 


$$
\left(\frac{\Delta I}{I}\right)_{T C}=\beta_{P} \cdot \Delta P
$$

The left hand side of the Eqn. 3.2 is the normalized deviation of the temperature corrected particle density, $\Delta \mathrm{P}$ is the pressure deviation from its mean value and $\beta_{P}$ is the pressure coefficient. The mean pressure coefficient value for all the detectors is found to be $-0.710 \pm 0.005 \% / h P a$ as shown in Fig. 4b. By using the mean pressure coefficient, the temperature corrected particle density data was corrected for pressure effect also. Fig. 5 displays a time series data for particle density over a period of one week, the top panel(a) shows the uncorrected particle density data variation and the bottom panel plot (b) shows the post atmospheric effect corrected particle density. A large reduction in the periodicity can been seen clearly particle density after applying the atmospheric corrections.

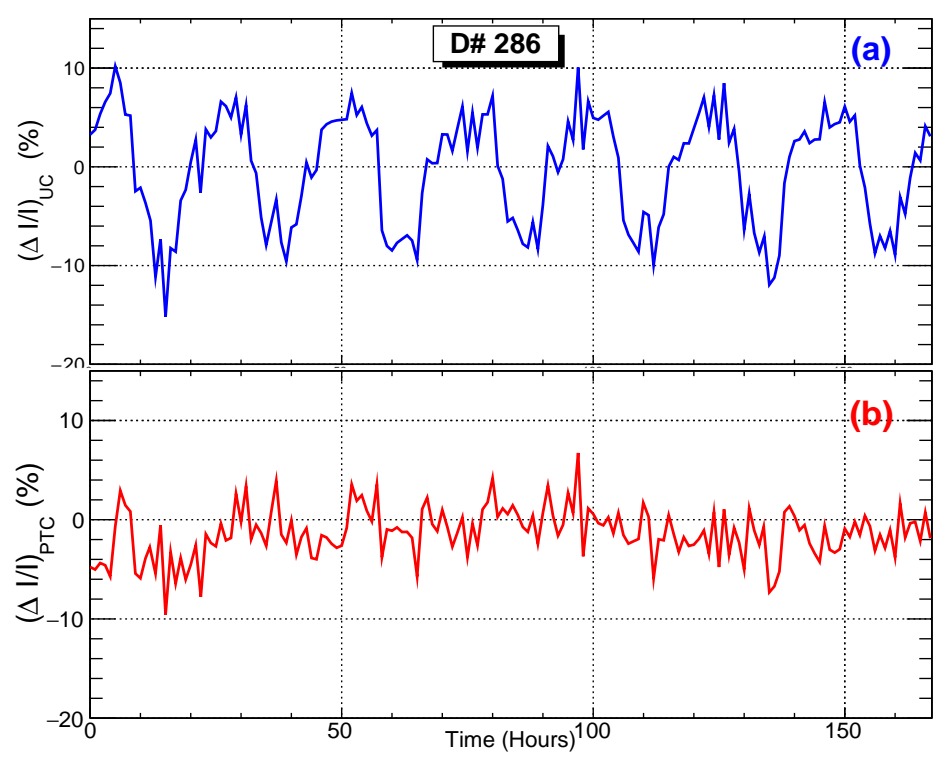

Figure 5: Particle density (\%) variation before (a) and after correction (b)

\section{Atmospheric effects on trigger rate}

In Fig. 6a, the trigger rate variation can be seen over a period of one week which indicates the presence of periodicity which later confirmed by applying the FFT on full year data. The power spectrum in Fig. $6 \mathrm{~b}$ shows the two dominant peaks at $1 \mathrm{cpd}$ and $2 \mathrm{cpd}$. In the analogy of particle density the presence of the periodicity in trigger rate can also be relate with the atmospheric effects as discussed in section 3. The values of temperature and pressure coefficients have been also determined for trigger rate which is found to be $-0.392 \pm 0.002 \% /{ }^{\circ} \mathrm{C}$ and $-0.597 \pm 0.008 \% / \mathrm{hPa}$ respectively. However, the correction of trigger rate is unrealistic due to number of showers are already recored. Thus, the effects can not be corrected. However, the corrections can be applied at final flux measurements. 

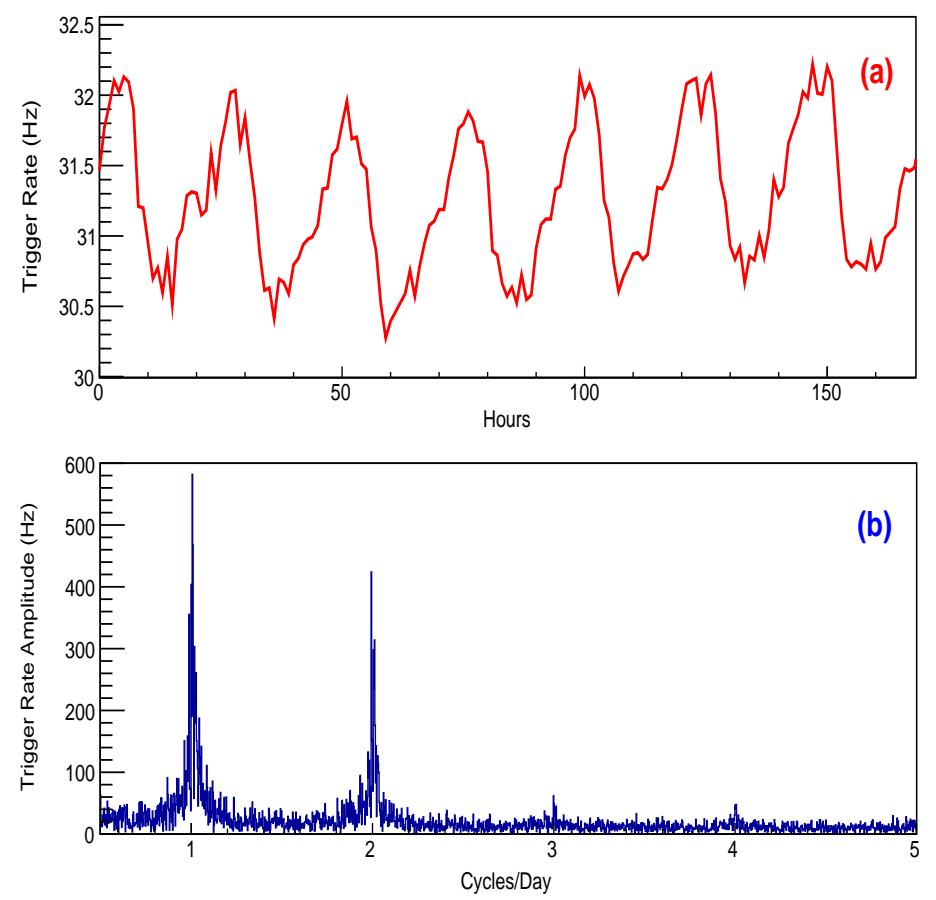

Figure 6: Trigger rate variation over a period of one week (a) and power spectrum of trigger rate for one year (b)

\section{Discussions}

The effects of atmospheric pressure and temperature on particle density as well as trigger rate has been described in details in the previous sections. Both the data show the significant dependence on the atmospheric parameters. The particle density shows the time lag with temperature due to thermal insulation of the plastic scintillation detectors as well as the temperature measurement at a single place. The mean temperature coefficient was calculated and found to be $-0.65 \pm$ $0.01 \% /{ }^{\circ} \mathrm{C}$ for all detectors after lag correction. Similarly, temperature corrected particle density shows strong anti-correlation with the atmospheric pressure. The mean pressure coefficient was calculated to $-0.710 \pm 0.005 \% / h P a$. Which expectedly comes very similar for all the detectors with $0.11 \mathrm{rms}$. The measurements show fairly good agreement with previous reports [6,7]. After correcting for atmospheric effects, the reduction in daily variations can be seen easily in Fig. $5 \mathrm{~b}$.

The atmospheric effects on GRAPES-3 trigger rate have also been observed. The temperature and pressure coefficients for trigger rate was calculated to be $(-0.392 \pm 0.002) \% /{ }^{\circ} \mathrm{C}$ and $(-0.597 \pm 0.008) \% / h P a$ respectively.

\section{Acknowledgement}

We thank D.B. Arjunan and K. Manjunath for the testing, installation and operation of various detector and electronic modules. We also thank S. Negi for his contribution in pressure calibration. 


\section{References}

[1] De Mendonca, et al., Journal of Geophysical Research: Space Physics, vol. 118 (2013) 23.

[2] Lindzen, Richard S. and Chapman, Space Science Reviews, vol. 10

[3] P.K. Mohanty, et al., Astroparticle Physics, vol. 79 (2016) 23.

[4] S.K. Gupta, et al., Nuclear Instruments and Methods in Physics Research A, vol. 540 (2005) 311.

[5] A.T. Young, Appl. Opt., vol. 2 (1963) 51.

[6] W.R. Leo, Techniques for Nuclear and Particle Physics Experiments, 1994, Springer.

[7] A. Daudin and J. Daudin, Journal of Atmospheric and Terrestrial Physics, vol. 3 (1953) 245.

[8] Y. Hayashi, et al., Nuclear Instruments and Methods in Physics Research A, vol. 545 (2005) 643.

[9] P.K. Mohanty, et al., Astroparticle Physics, vol. 31 (2009) 24.

[10] P.K. Mohanty, et al., Pramana, vol. 81 (2013) 343.

[11] http://www.et-enterprises.com/photomultipliers 